\title{
Phonon dispersion of oriented DNA by inelastic x-ray scattering
}

\author{
M. Krisch, ${ }^{1}$ A. Mermet, ${ }^{1}$ H. Grimm, ${ }^{2}$ V. T. Forsyth,${ }^{3,4}$ and A. Rupprecht ${ }^{5}$ \\ ${ }^{1}$ European Synchrotron Radiation Facility, Bô̂te Postale 220, F-38043 Grenoble Cedex, France \\ ${ }^{2}$ Institut für Festkörperforschung, Forschungszentrum, D-52425 Jülich, Germany \\ ${ }^{3}$ Partnership for Structural Biology, clo Institute Laue-Langevin, Bôte Postale 156, F-38043 Grenoble Cedex, France \\ ${ }^{4}$ Institute for Science and Technology in Medicine, Keele University Medical School, Staffordshire, ST4 7QB, England \\ ${ }^{5}$ Division of Physical Chemistry, Arrhenius Laboratory, University of Stockholm, S-106 91, Stockholm, Sweden
}

(Received 12 October 2005; published 16 June 2006)

Films of oriented deoxyribonucleic acid (DNA), prepared by the wet spinning method, have been studied using inelastic $\mathrm{x}$-ray scattering. Spectra were recorded within the range of energy transfers $-30<\hbar \omega<30 \mathrm{meV}$ at momentum transfers $\hbar \mathbf{Q}$ ranging from 2.5 to $30 \mathrm{~nm}^{-1}$ whereby the direction of Q essentially coincided with the helical axis. Measurements at ambient temperature cover samples in the $A, B$, $C$, and $D$ conformations of DNA. Within the limits of the instrumental resolution, the spectra were analyzed by the response of a damped harmonic oscillator delivering dispersion and damping of modes having displacements with nonzero projections onto $\mathbf{Q}$, i.e., essentially the compression waves traveling along the helical axis. The longitudinal speed of sound resulting from the sinusoidal dispersion varies only weakly with conformation. Our sound speed values are compared to results from Brillouin spectroscopy. The dispersion curves exhibit a minimum at about the inverse rise per residue, which—-together with strong elastic scattering-reflect the large degree of disorder. Overdamping of the modes is observed for $Q>5 \mathrm{~nm}^{-1}$. The possibility that the observed large damping parameters are due to several contributing modes is discussed in terms of a simple model calculation for an idealized double helix. Whereas the quasicrystalline approximation for an effective disordered chain could well describe the sinusoidal dispersion, it fails to reproduce the observed damping by one order of magnitude. Our results indicate that the high-frequency dynamics of DNA is liquidlike and is most appropriately described by instantaneous normal modes of short correlation length.

DOI: 10.1103/PhysRevE.73.061909

PACS number(s): 87.15. $-\mathrm{v}, 78.70 . \mathrm{Ck}, 63.50 .+\mathrm{x}$

\section{INTRODUCTION}

Double-stranded DNA can adopt one of five distinct conformations depending on base-pair sequence, hydration, and ionic conditions. The $A$ (11 base-pairs per helix pitch of $28.2 \AA), B$ (10 base-pairs per helix pitch of $33.4 \AA)$, and $C$ conformations (9.3 base-pairs per pitch of $31.0 \AA$ ) are found in natural "random sequence" DNA [1-3]. Two other conformations, known as $D$ (8 base-pairs per helix pitch of $24.2 \AA)$ and $Z$ (12 base-pairs per helix pitch of $45.0 \AA$ ), are observed in DNA having highly repetitive sequences $[4,5]$. Of the five structures, the $A, B, C$, and $D$ conformations are right handed helices, and the $Z$ conformation is left handed. With the exception of the $Z$ conformation, all of these structures were determined by $x$-ray fiber diffraction [1-3]. The $Z$ conformation was solved by x-ray crystallography [6] and then later described in fibrous samples by Arnott et al. [5]. All five conformations can be observed in fibers of polymeric DNA, whereas only the $A, B$, and $Z$ structures have been observed in oligonucleotide single crystals. Structural transitions between these forms are possible as a function of hydration and ionic strength, and with suitable humidity control [7]. Such changes can be studied in real time by time-resolved $\mathrm{x}$-ray fiber diffraction [8]. The biological significance of the different conformations of DNA is not clear. However it would be rather surprising if the ability of the double helix to adopt these unique structures were not exploited in biological function.

Knowledge of the dynamical properties of DNA is of importance for developing theoretical models of biological processes such as base pair opening, replication, and transcription. For example, an energetically intriguing puzzle is the formation of about 10-20 open base pairs in the complex of DNA with ribonucleic acid polymerase as a result of a minor temperature increase [9]. Therefore, numerous studies have focused on the low frequency spectra of DNA, which reflect the forces stabilizing the double helix. The very existence of the various conformations and of transitions between these [10] underlines the subtle balance of the relevant interactions between DNA, counterions, hydration, and excess salt. In order to shed light on the transition from $A$ to $B$ conformation at around $90 \%$ relative humidity (RH), Prohofsky and co-workers [11] applied the soft mode concept [12] to DNA by variation of the effective dielectric constant and/or the polarizability in their model on the atomistic level. The temperature dependence was incorporated in this model by a modified self-consistent phonon approximation (MSPA), adopting the Morse potential for the hydrogen bonds linking the two strands of DNA. This allowed the study of DNA melting, i.e., disruption of the double helix, as a function of base pair composition $[13,14]$. Within this framework the DNA is represented by an effective harmonic lattice with corresponding phononlike excitations. On the other hand, (i) native DNA is intrinsically not translationally invariant since the base-pair sequence varies along its length, (ii) short range order and mixtures containing more than one conformation can be identified in fiber diffraction patterns, and (iii) hydrogen exchange experiments suggest dynamical local opening of the double helix well below the denaturation temperature. Thus apart from small oscillations, both the influence of conformational disorder and the consideration of large amplitude fluctuations exploring the anharmonicity of the interaction landscape are expected to play an important role in modeling DNA dynamics $[15,16]$. This has led Peyrard, Bishop, and 
co-workers to develop a model exhibiting breatherlike excitations, which provide a means to focus thermal energy in space $[17,18]$. Attention is paid to one degree of freedom per base pair only, i.e., the hydrogen bond stretching governed by the Morse potential. This model has been extended to include the rotation of base pairs around the helical axis due to the inherent connection between local unwinding and base pair opening [19]. In order to incorporate the aspects mentioned above, the concept of instantaneous modes [20] has been applied in order to obtain the short time dynamics of DNA using a replicated transfer matrix formalism. A major finding is that normal modes are highly disordered and coherent over a few base pairs only. Thus, there are two approaches in modeling the low frequency dynamics of DNA-either exploiting translational invariance (solidlike, plane waves) or exploiting one-dimensionality by using a transfer matrix (liquidlike, instantaneous modes).

Experimental attempts to determine the dynamical properties of DNA have been mostly performed by Brillouin [21-24] and Raman scattering [25]. The sound velocity parallel and perpendicular to the helix axis for the $A$ and $B$ conformation as a function of humidity content was determined, and a decrease of both velocities with increasing humidity, as well as slight differences in the sound attenuation between $A$ and $B$ conformation were observed. Temperaturedependent Brillouin scattering studies revealed an increase of the sound speed and a decrease of the sound attenuation with decreasing temperature. Raman scattering results demonstrate a stiffening of the lowest lying mode by almost a factor two between $0{ }^{\circ} \mathrm{C}$ and $-50^{\circ} \mathrm{C}$, whereas between $-50{ }^{\circ} \mathrm{C}$ and $-150^{\circ} \mathrm{C}$ the mode is almost frequency independent. These results indicate the presence of two relaxation processes in hydrated DNA, associated with the coupling of the collective vibrational modes with the primary and the second hydration shell. At room temperature the relaxation times amount to $\tau_{1}=4 \times 10^{-11} \mathrm{~s}$ and $\tau_{2}=2 \times 10^{-12} \mathrm{~s}$ (first and second hydration shell, respectively).

The dynamics of DNA approaching length scales associated with its structural periodicity, i.e., the pitch of the double helix, was studied by coherent inelastic neutron scattering (INS) $[26,27]$. Coherent INS, as well as inelastic x-ray scattering (IXS) techniques, directly probe the dynamic structure factor $S(Q, \hbar \omega)$, which is the Fourier transform in space and time of the density-density correlation function [28]. Here, $\hbar \omega$ is the energy of the excitation, associated with density fluctuations induced by the collective movement of the particles. In the macroscopic time-space domain, these excitations, known as sound waves, display a linear dispersion. In the mesoscopic regime, accessed by INS and IXS, typical length and time scales are comparable to interparticle distances and correlations and the lifetime of these correlations, respectively. Due to the kinematic limitations of INS, the experiment could not be performed at small momentum transfers within the first Brillouin zone [corresponding to momentum transfer values up to $Q_{\max } / 2$, where $Q_{\max }$ marks the position of the first peak in the static structure factor $S(Q)$ ] [29]. The experiment was instead performed in the vicinity of the structure factor maximum. Utilizing an effective hyperbolic dispersion relation a sound speed of $2180 \mathrm{~m} / \mathrm{s}$ could be derived for $B$ DNA at room temperature.
Very recently, a pioneering IXS experiment was performed on shear-aligned columnar hexagonal liquid crystalline $B$ DNA dispersed in water and in $0.085 \mathrm{M} \mathrm{MgCl}_{2}$ [30]. As a matter of fact, within the present context IXS is the spectroscopic tool of choice for various reasons: (i) In contrast to coherent INS, IXS is free of kinematic limitations, and therefore collective excitations in the first Brillouin zone can be assessed without compromise in the energy resolution; (ii) the IXS process is predominantly coherent, whereas for INS the incoherent contributions have to be evaluated by reference scans [26,31]. This method breaks down for small $Q$-values due to the lateral ordering of the DNA fibres; (iii) the necessary sample volume for INS amounts to approximately $1.5 \mathrm{~cm}^{3}$, whereas IXS needs only $1 \%$ of this value. This small volume opens the possibility to investigate as well artificial DNA. The IXS results spanned the $2-30 \mathrm{~nm}^{-1}$ momentum transfer range, and were analyzed within the framework of the generalized three effective eigenmode (GTEE) theory [32]. An oscillatory behavior of the phonon dispersion was observed with a linear increase at low $Q$ (sound velocity of about $3100 \mathrm{~m} / \mathrm{s}$ ), a maximum at about $9 \mathrm{~nm}^{-1}$, no detectable inelastic features in the $Q$ range around the position of the maximum in the $S(Q)$, and a second increase of the phonon energy for $Q>22 \mathrm{~nm}^{-1}$. These results are in variance to previous INS results where the phonon energies do not go down to zero, which was reported to be due to anticrossing with low-lying optical modes [26]. These discrepancies may be due to the different methods of data analysis, and underline the importance of further experiments.

Here we present an IXS study on the dynamics of oriented DNA films for four different conformations at room temperature in the 2.5 to $30 \mathrm{~nm}^{-1}$ momentum transfer range. Despite significant structural differences, the lattice dynamics are only weakly affected. Overdamping of the modes is observed for $Q>5 \mathrm{~nm}^{-1}$, and its origin discussed by comparing our experimental results with two model calculations, namely (i) an idealized double helix with six dispersion curves, corresponding to the six degrees of freedom, and (ii) a onedimensional disordered linear chain. While the limited experimental resolution does not allow resolving individual modes at low $Q$, the resulting dispersion is qualitatively well reproduced, and provides a natural explanation for the minimum in the sinusoidal dispersion at momentum transfers in the vicinity of the first structure factor maximum. The disordered chain mode provides a good description of the phonon dispersion as well, but underestimates the amount of phonon damping by one order of magnitude.

The paper is organized as follows. Section II provides the experimental details, including sample preparation and characterization as well as the experimental setup, the theoretical formalism and the fitting procedure. The results on $B$ DNA are presented and discussed in Sec. III, while Sec. IV provides the comparison with the other three conformations and the discussion. Section V summarizes the main results of the paper.

\section{EXPERIMENTAL DETAILS}

\section{A. Sample preparation and characterization}

Oriented samples in the four conformations $A, B, C$, and $D$ DNA with $11,10,28 / 3$, and 8 base pairs per turn of the 
helix, respectively, have been prepared. Material for the $A$ and $B$ DNA sample was extracted from salmon testes (FLUKA Chemie AG) and for the $C$ DNA sample from calf-thymus (Worthington Biochem. Corp.) - both purchased as sodium salts. The $D$ DNA sample was made from a highly polymerized sample of the DNA polynucleotide poly $[d(A-T)] \cdot \operatorname{poly}[d(A-T)]$. This polymer was made by enzymatic synthesis by incubation of DNA polymerase $I$ with equimolar quantities of $d A T P$ and $d T T P$, with a small amount of primer. Procedures for doing this are described by Pope $e t$ al. [33]. The purified polynucleotide was prepared as the potassium salt.

In order to stabilize the $B$ or the $C$ conformations, the $\mathrm{Na}^{+}$ counterions were exchanged against $\mathrm{Li}^{+}$by dialysis. From aqueous solutions of $\mathrm{Na}$ and $\mathrm{Li}$ DNA, respectively $(\sim 1 \mathrm{mg} / \mathrm{ml})$, highly oriented DNA films were produced by the wet-spinning method [34]. In this method, a motorized syringe extrudes the DNA solution through a spinneret (60 $\mu \mathrm{m}$ holes) into a column $(\sim 1 \mathrm{~m})$ of an ethanol-water solution, causing precipitation. The resulting DNA fibers are continuously stretched by winding them up on a rotating Teflon-coated glass cylinder after passing through a $V$-shaped guide. A slow adapted axial motion of the cylinder between adjustable limits allows for winding up of parallel fibres layer by layer. The conditions for spinning the synthetic poly $[d(A-T)]$. poly $[d(A-T)]$ were somewhat different. Firstly, $\mathrm{KF}$ was used as electrolyte as opposed to the $\mathrm{NaCl}$ or $\mathrm{LiCl}$ used for the $A$ DNA and $B$ DNA samples respectively; second, the polynucleotide concentration of the spinning solution was about three times higher to compensate for the lower degree of polymerization.

The spun deposits were subsequently bathed in an aqueous ethanol solution of the required electrolyte concentration [35]. After removal from this solution, the excess liquid was removed and the cylinder with spun deposit placed in a desiccator (in a refrigerator). This resulted in slow drying as ethanol or water condensed on the desiccator walls. As a result, the fibers fuse together forming an oriented DNA film that was then cut cleanly from the cylinder. Adding a small amount of silica gel in the desiccator speeded up this drying procedure. The films obtained were stored above a saturated $\mathrm{NaClO}_{3}$ solution, maintaining a relative humidity of $75 \%$. The asymptotic water uptake of the DNA film corresponds to about $15 \mathrm{H}_{2} \mathrm{O}$ per base pair. This corresponds to about optimum conditions for the correlation length along the helix [36]. The excess salt content of the films is about $0.12 \mathrm{NaCl}$ /base pair for the $A, 0.70 \mathrm{LiCl} /$ base pair for the $B, 0.06 \mathrm{LiCl}$ /base pair for the $C$, and $0.5 \mathrm{KF} / \mathrm{base}$ pair for the $D$ DNA sample.

The DNA conformations were verified by recording x-ray fiber diffraction patterns from test pieces. In order to maintain the humidity during exposure $(\sim 20 \mathrm{~min})$, the films were placed between thin mica sheets $(0.12 \mathrm{~mm})$. Tightening was achieved by high vacuum grease whereby a sideways displaced "chamber" was formed for the added droplet of saturated salt solution. The observed loss of weight was only $1 \%$ per day. The resulting patterns are shown in Fig. 1. Some small sharp spots can be seen in these pictures. These arise from the mica sheets. The patterns are typical for the various
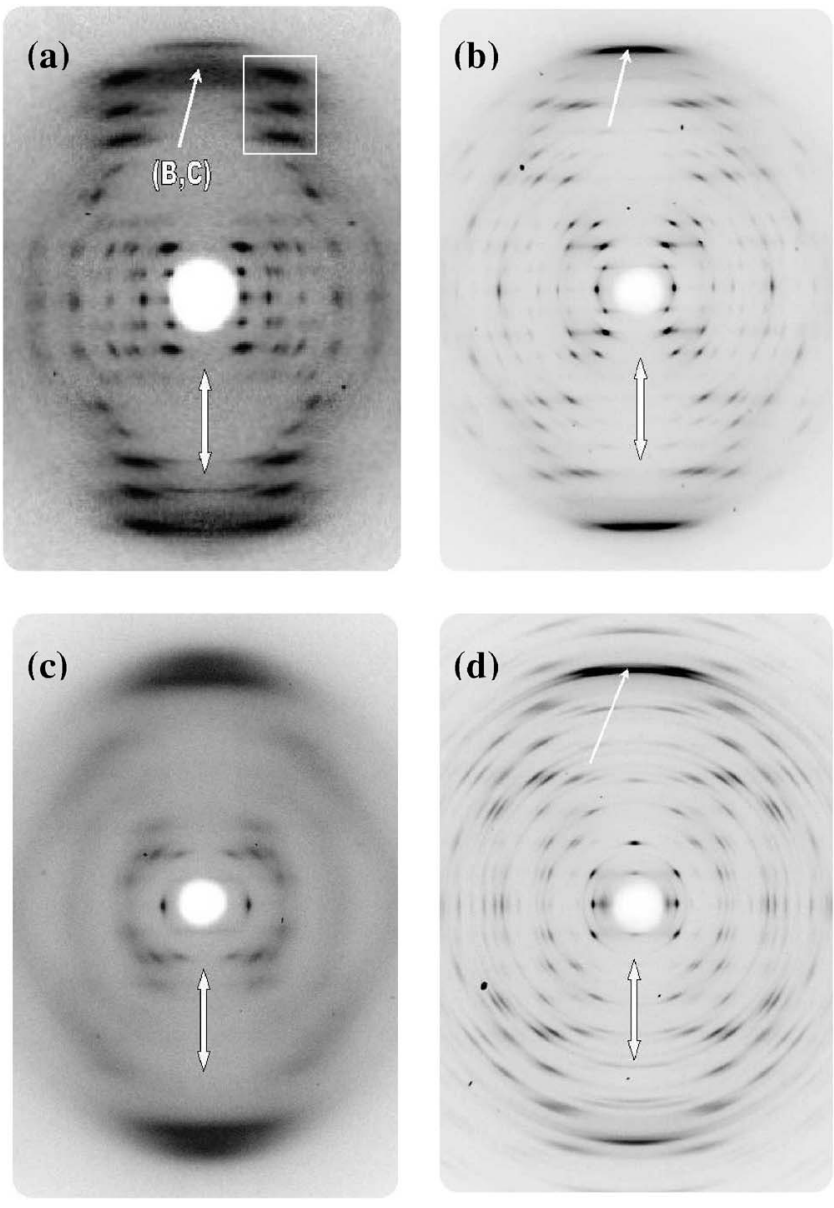

FIG. 1. Fiber diffraction pattern of the DNA samples. (a) $A$ DNA. The double arrow indicates the helical axis. The white rectangle emphasizes intense features in the $6^{\text {th }}, 7^{\text {th }}$, and $8^{\text {th }}$ layer line which are characteristic for the $A$ conformation. The arrow marks contamination by the $B$, and $C$ conformation. (b) $B$ DNA. The $10^{\text {th }}$ layer line (base pair peak) is also marked, corresponding to the inverse distance of base pairs along the helical axis (double arrow). (c) $C$ DNA sample. (d) $D$ DNA. The inclined arrow marks contamination by the $B$ conformation close to the $7^{\text {th }}$ layer line.

conformations and exhibit good orientation. As expected, the worst in this respect is the D DNA sample [Fig. 1(d)], because of the lower molecular weight of the synthetic poly $[d(A-T)]$. poly $[d(A-T)]$. The reduced length of the molecules diminishes their response to the orienting strain field built up by stretching and winding up the bundle of fibers during the spinning process. The sample closest to "translational invariance" is the $B$ DNA sample for which the base pairs are moderately inclined $\left(\Theta=6.3^{\circ}\right)$ and their center of mass is close to the helical axis $(d=0.03 \mathrm{~nm})$. The $C$ conformation is similar $\left(\Theta=8^{\circ}, d=0.05 \mathrm{~nm}\right)$, however, with a much lower correlation length than for $B$ as evidenced by the large width of the base stacking peak [Fig. 1(c)]. The form factor of the inclined base pairs $\left(\Theta=20.3^{\circ}, d=0.49 \mathrm{~nm}\right)$ in the $A$ conformation leads to the characteristic intense off-center signals in the $6^{\text {th }}, 7^{\text {th }}$, and $8^{\text {th }}$ layer line indicated in Fig. 1. However, the intensity bridging those off-center features of the $8^{\text {th }}$ layer line is incommensurate and due to much less inclined base pairs, i.e., a contamination with $B$ DNA [37]. A 


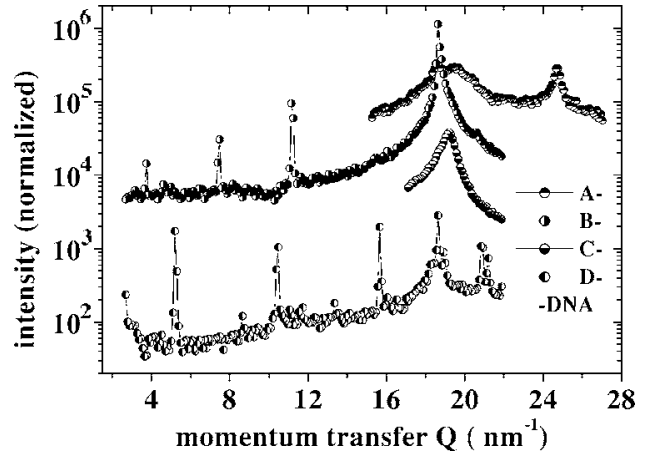

FIG. 2. Elastic scans along the helical axis for the four investigated conformations. The scans represent corresponding sections through the fiber diffraction patterns shown in Fig. 1. They are displaced vertically by a constant amount for clarity. The peak positions are discussed in the text.

similar contamination by $B$ DNA is recognizable for the D DNA sample [Fig. 1(d)] because of the displacement of the $7^{\text {th }}$ layer line by about $2.4 \%$ from the regular position.

The in situ characterization and structural control of the samples was done by elastic scans along the helical axis. Figure 2 shows typical scans for the four conformations. The peak due to the $11^{\text {th }}$ layer line at $Q=24.6 \mathrm{~nm}^{-1}$-corresponding to a helical pitch of $2.81 \mathrm{~nm}$ - was taken as characteristic for the $A$ conformation. Its relatively weak intensity is due to the inclined base pairs. One recognizes again the $B, C$ contamination by the broad peaks between 18.5 and $19.5 \mathrm{~nm}^{-1}$. Note that these peaks are by an order of magnitude smaller than those from the corresponding pure conformations. This puts the estimate for the contamination in the $10 \%$ region. The characteristic feature for the $B$ conformation is the strong base stacking peak at $Q=18.6 \mathrm{~nm}^{-1}\left(10^{\text {th }}\right.$ layer line, helical pitch $=3.38 \mathrm{~nm}$ ). The signals from the $2^{\text {nd }}, 4^{\text {th }}$, and $6^{\text {th }}$ layer line are also visible. The base stacking peak for the $C$ conformation is shifted to $Q=19.2 \mathrm{~nm}^{-1}$ (helical pitch $=3.05 \mathrm{~nm}$ ). For the $D$ conformation one recognizes the peaks due to the $2^{\text {nd }}$, $4^{\text {th }}, 6^{\text {th }}, 7^{\text {th }}$, and $8^{\text {th }}$ layer lines. Closer inspection reveals that the $2^{\text {nd }}, 4^{\text {th }}$, and $6^{\text {th }}$ layer lines follow closely the helical pitch of $2.41 \mathrm{~nm}$, whereas the $7^{\text {th }}$ layer line is slightly shifted towards the commensurate base stacking peak of $B$ DNA. The $8^{\text {th }}$ layer line exhibits already a double peak shape indicating some admixture of the $B$ conformation. In summary, although the fiber diffraction patterns are found to be characteristic for the expected conformations, one has to keep in mind that the conformational homogeneity is limited.

\section{B. Inelastic x-ray scattering setup}

The experiment was carried out at the Inelastic X-ray Scattering Beamline II (ID28) at the European Synchrotron Radiation Facility in Grenoble, France. The x rays from the undulator source are monochromated by a cryogenically cooled silicon (111) double crystal monochromator and a high-energy resolution backscattering silicon monochromator, operating at a Bragg angle of $89.98^{\circ}$, and utilizing the (11 11 11) reflection order. The focusing is accomplished by a gold-coated toroidal mirror, which provides a focal spot at the sample position of 270 (horizontal) $\times 80$ (vertical) $\mu \mathrm{m}^{2}$ FWHM. The scattered photons are energy analyzed by a Rowland circle five-crystal spectrometer of $6.5 \mathrm{~m}$ radius, operating at the same reflection order as the monochromator. The energy-analyzed photons are detected by a Peltiercooled silicon diode detector. The momentum transfer $Q=2 k_{i} \sin \left(\theta_{s} / 2\right)$, where $k_{i}$ is the incident photon wave vector and $\theta_{s}$ is the scattering angle, is selected by rotating the spectrometer around a vertical axis passing through the scattering sample in the horizontal plane. Since there are five independent analyser systems, spectra at five different momentum transfers can be recorded simultaneously. Their separation is energy dependent and for the silicon (11 11 11) reflection, utilized in the present experiment, it amounts to $3 \mathrm{~nm}^{-1}$. The energy scans were performed varying the monochromator temperature, while the analyzer temperature is kept fixed. Conversion from the temperature scale to the energy scale is accomplished by the following relation: $\Delta E / E=\alpha \Delta T$, where $\alpha=2.58 \times 10^{-6}$ is the linear thermal expansion coefficient of silicon at room temperature. The validity of this conversion has been checked by comparing the measured diamond dispersion curve for longitudinal acoustic and optical phonons with well established inelastic neutron scattering results. The overall experimental resolution is determined by measuring the scattering from a disordered sample of plexiglass at a $Q$ transfer of $10 \mathrm{~nm}^{-1}$, corresponding to the first maximum in the static structure factor $S(Q)$, and at $T=10 \mathrm{~K}$ in order to maximize the elastic contribution to the scattering. Further details on the instrument and the IXS technique can be found elsewhere [38-42].

For each of the sample types, two pieces of DNA film with dimensions of $8 \times 8 \times 0.12 \mathrm{~mm}^{3}$ were used for the inelastic scattering experiment. These were sandwiched between two $\mathrm{Nb}$ cubes of $8.5 \mathrm{~mm}$ length. This arrangement was then inserted into a sample cell made of copper and having four diamond windows allowing for passage of the beam between the $\mathrm{Nb}$ cubes either parallel or perpendicular to the fiber axis. Apart from a test scan, the fiber axis was aligned approximately parallel to the momentum transfer vector $Q$. Sealing the cell at its bottom cover activated a spring keeping the slit between the $\mathrm{Nb}$ cubes centered with respect to the diamond windows which had a free diameter of about $3.5 \mathrm{~mm}$. The cell was then fixed to the $\mathrm{Cu}$ plate of a closed cycle refrigerator. Helium gas, humidified to the required level for the particular DNA sample being studied, was passed continuously through the sample cell. The humidity of the outgoing gas was monitored $(66 \% \mathrm{RH}$ for $D$ DNA, 75\% RH else).

After alignment of the sample in the scattering plane, data acquisition consisted in alternatively taking elastic scans testing the structural quality of the sample, and recording five constant $Q$ scans simultaneously in the range $-30 \mathrm{meV}<\hbar \omega<+30 \mathrm{meV}$. IXS spectra were recorded for three settings for each conformation covering the range $2.5 \mathrm{~nm}^{-1}<Q<30 \mathrm{~nm}^{-1}$ corresponding to scattering angles $1.3^{\circ}<\Theta_{s}<15.6^{\circ}$. This entails a maximum angle of $7.8^{\circ}$ between the fiber axis and the momentum transfer $\mathbf{Q}$. Based on constant- $Q$ scans taken with neutrons at positions slightly off from the helical axis, this deviation is not expected to alter 
the response seriously. The structural quality of the sample was checked by $S(Q)$ scans before and after each IXS scan, and the sample was shifted laterally by $0.5 \mathrm{~mm}$ in order to avoid radiation damage. The inelastic data were collected within two counting intervals of about $6 \mathrm{~h}$ for each of the three settings of the analyzers.

\section{Fit procedure}

In view of several contributing factors to the strong scattering signal, centered around zero energy transfer, and the limited energy resolution, we have restricted the description of the IXS signal to two types of response, i.e., an effective Lorentzian for the strong (quasi-)elastic scattering and a "damped harmonic oscillator" ansatz (DHO) representing the resolvable vibrational part. The choice of a DHO function has several motivations: (i) this line shape can be derived using a Markovian ansatz for the memory function, for which one assumes that the time scale of the considered density fluctuations, and thus the reaction of the surrounding medium, is much faster than the relaxation time associated with any relaxation process active in the system. This corresponds to a model of the memory function entering in the generalized Langevin equation made up of a constant and a $\delta$ function; (ii) the DHO model is routinely used in the analysis of neutron data and MD simulations of disordered systems; (iii) it is a model function utilizing a minimum of parameters, and serves therefore to highlight the main features of the spectra without the need to invoke a specific theory. The shape of the resolution is essentially Lorentzian as shown e.g. in Fig. 3 for small values of $Q$ where the quasielastic broadening is negligible. The full width at half maximum (FWHM) ranges from 1.7-1.9 meV for the five analyzers.

The spectral response of the DHO function with frequency $\omega_{o}$ and damping $\Gamma$ is described by

$$
\chi^{\prime \prime}(\omega)=\left|F_{\text {dyn }}\right|^{2} \frac{\omega \Gamma}{\left(\omega^{2}-\omega_{0}^{2}\right)^{2}+\omega^{2} \Gamma^{2}}=\pi \frac{S_{B}(\omega)}{n(\beta \omega)+1},
$$

where $\chi^{\prime \prime}(\omega)$ denotes the imaginary part of the susceptibility and $S_{B}(\omega)$ the balanced scattering function. $n(x)$ represents the Bose occupation factor $\left(e^{x}-1\right)^{-1}$ and $\beta=1 / k_{B} T$. Since we consider one effective oscillator, only, the modulus of the dynamical structure factor $F_{\text {dyn }}$ may be considered as a $Q$-dependent scale factor. Thus, the experimental data are described by seven parameters: one for a constant background, one for the precise center of the abscissa, two for the central Lorentzian (height and width), and three (height, energy position, and width) for the DHO. In order to minimize as much as possible parameter correlation, we exploited the possibility to reduce the number of parameters for each step of the parametrization. First, the determination of the background was decoupled from the fit of the ansatz by the requirement of detailed balance. Suitable basis for this determination were the energy windows $25 \mathrm{meV}$ $<|\omega|<30 \mathrm{meV}$, delivering a statistical error of about $30 \%$. Second, the measured data were normalized by their maximum intensity, cast into the $\left|\chi^{\prime \prime}(\omega)\right|$ representation and averaged over energy gain and energy loss. Initial parameters were estimated for width and amplitude of the effective
Lorentzian describing the strong central scattering. The DHO response was isolated by subtraction of the resolution folded Lorentzian and fitted by the three corresponding parameters. A few iterations of this decoupling scheme led to selfconsistency between those two groups of parameters even for the critical $Q$ ranges in the neighborhood of the base stacking peak at $Q=18.6 \mathrm{~nm}^{-1}$, and at small $Q$ values where the oscillator contribution is relatively small.

\section{RESULTS FOR $B$ DNA}

\section{A. IXS spectra and dispersion relation}

Figure 3 shows the IXS spectra of $B$ DNA, together with their best fit results and the corresponding individual components. We note that the DHO model provides an excellent description of the experimental spectra. Inelastic features with an increasing width are recognizable throughout the spanned $Q$ range with exception of the region around $Q$ $=18.6 \mathrm{~nm}^{-1}$ where the spectrum is governed by strong (quasi-)elastic scattering. For $Q$ values of 17.5 and $20.52 \mathrm{~nm}^{-1}$, the inelastic features appear centered around zero energy transfer, while the fit results in a finite excitation energy. This is a consequence of the DHO model function. For momentum transfers $Q>20.52 \mathrm{~nm}^{-1}$, the inelastic excitations are becoming increasingly overdamped. It is obvious from Fig. 3 that the IXS spectra are dominated by nonresolvable central and low energy scattering. Basically, three contributions have to be considered: (i) elastic scattering due to lack of translational invariance, (ii) relaxational motion, and (iii) low energy torsional modes. In fact, the development of a strong quasielastic component for $T>T_{g} \cong 200 \mathrm{~K}$ has been observed by neutron scattering in the region from $2 \mu \mathrm{eV}$ up to about $5 \mathrm{meV}$ [43]. This component includes relaxational motions of the water hull as well as conformational fluctuations ( $B \Leftrightarrow C$ conformation). Cooling of the sample below $T_{g}$ leads to a random freezing of those fluctuations and thus does not reduce this central scattering appreciably. The width of the effective central Lorentzian, as obtained from the fit to the IXS data, remains small as compared to the resolution, reaching a maximum of about $0.3 \mathrm{meV}$ for the largest $Q$. Within the presently achievable energy resolution a more refined analysis of this central component is therefore not possible.

The wave vector dependence of the inelastic features is shown in the top panel of Fig. 4, while the corresponding dependence of the excitation width is reported in the bottom panel. Coarsely speaking, the dispersion of the eigenfrequency $\omega_{0}$ resembles that for a linear chain with dominating next neighbor interaction subjected to disorder. This is reflected by the increase of the eigenfrequency for small $Q$ up to a maximum at about half of the base stacking peak at $Q_{0}=18.6 \mathrm{~nm}^{-1}$, followed by the decrease towards $Q_{0}$ where the mode becomes nonresolvable. Beyond $Q_{0}$, the mode reappears, however heavily overdamped. The sound velocity, propagating along the helix axis, can be derived from a linear fit to the low $Q$ part, and is determined to be $2830 \pm 50 \mathrm{~m} / \mathrm{s}$. This value is slightly larger than values obtained by Brillouin scattering: $2400 \mathrm{~m} / \mathrm{s}$ [23] and $2600 \mathrm{~m} / \mathrm{s}$ [24] and for $B$ DNA at $75 \%$ humidity. The higher sound speed, observed in the 


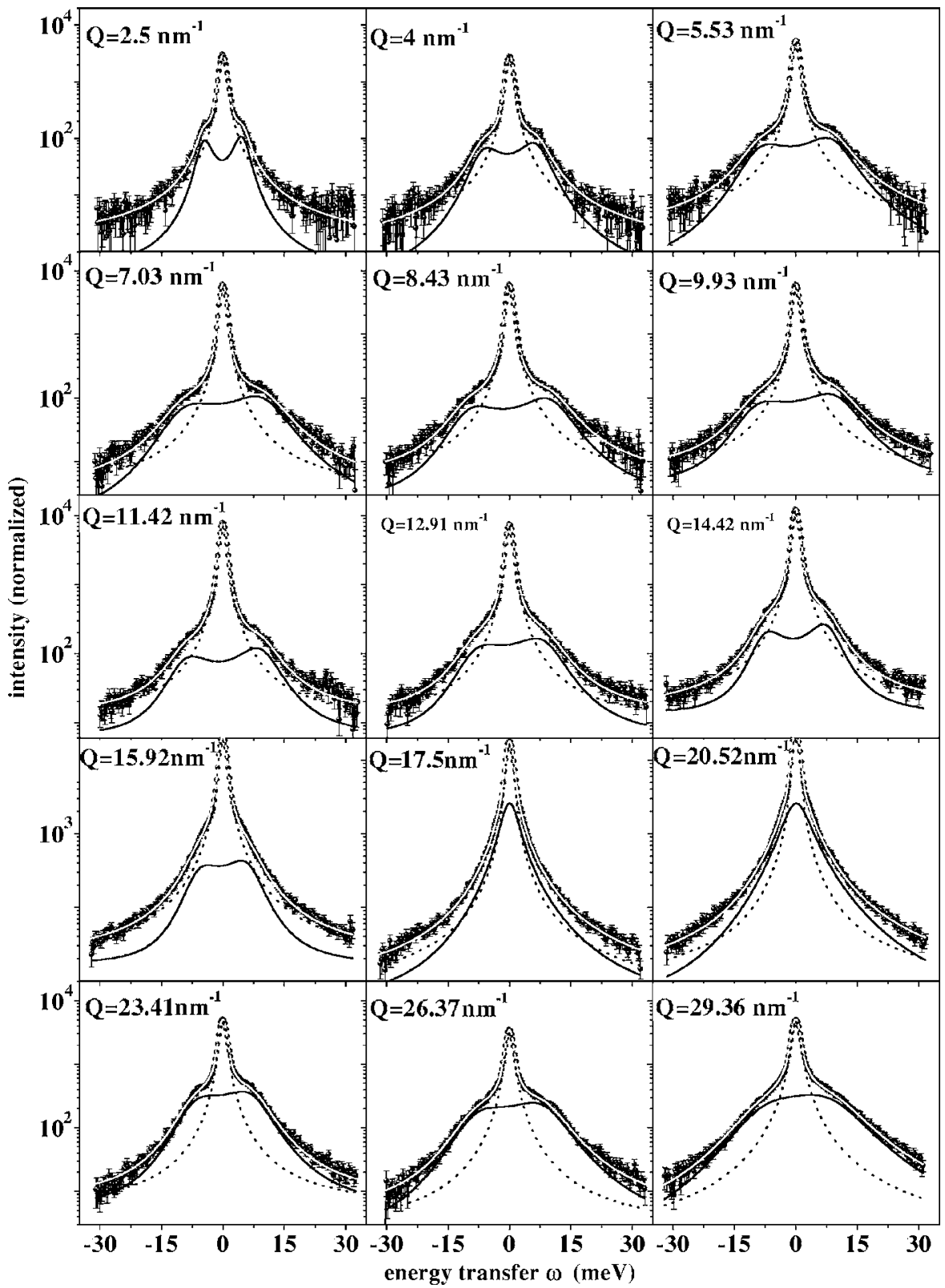

FIG. 3. Measured and fitted (white lines) constant- $Q$ scans for $B$ DNA at room temperature. The elastic and quasielastic part is indicated by the dashed curves, whereas the solid lines represent the oscillatory part. See text for details of the fitting procedure.
IXS experiment, resides in the difference of the probed wave vectors between Brillouin and inelastic x-ray scattering, and marks the difference between the viscous regime, characterized by the adiabatic sound velocity $c_{0}$, and the elastic regime with its infinite frequency sound velocity $c_{\infty}$. The previous INS results delivered, depending on the approach [(i) linear dispersion around $Q_{0}$, or (ii) use of an effective hyperbolic dispersion] $v=1850 \pm 50 \mathrm{~m} / \mathrm{s}$ and $2180 \pm 50 \mathrm{~m} / \mathrm{s}$, respectively, with a finite excitation energy of $1.7 \mathrm{meV}$ [26]. The lower sound velocity derived from these results is a direct consequence of the fact that the INS measurements could only be performed around $Q_{0}$, where the shape of $S(Q, \omega)$ is very sensitive to the effects of disorder and coupling of modes in the DNA chain.

The previous IXS study, using the generalized three effective eigenmode theory [30], yielded a value of zero for the phononlike modes at $Q$ values between 16 and $22 \mathrm{~nm}^{-1}$. This is not only in contrast to the present analysis, where in this $Q$ range the excitation energy never attains a value below $4 \mathrm{meV}$, but also to the INS results, which clearly identify inelastic features. In order to shed further light on this issue we performed two types of model calculations, which are described in detail below.

\section{B. Model calculations}

Several questions arise from the dispersion curves shown in Fig. 4. The most obvious one concerns the large values obtained for the damping parameter $\Gamma$ and the minimum in the phonon dispersion around $18.5 \mathrm{~nm}^{-1}$. The latter can be interpreted as a direct consequence of the disorder due to the variance in average distances and contamination with other conformations, which affects as well the damping parameter. On the other hand, it is not excluded that $\Gamma$ just represents an averaging over several modes. In this context, it might be instructive to inspect the predictions for frequency $\omega_{0}$ and 


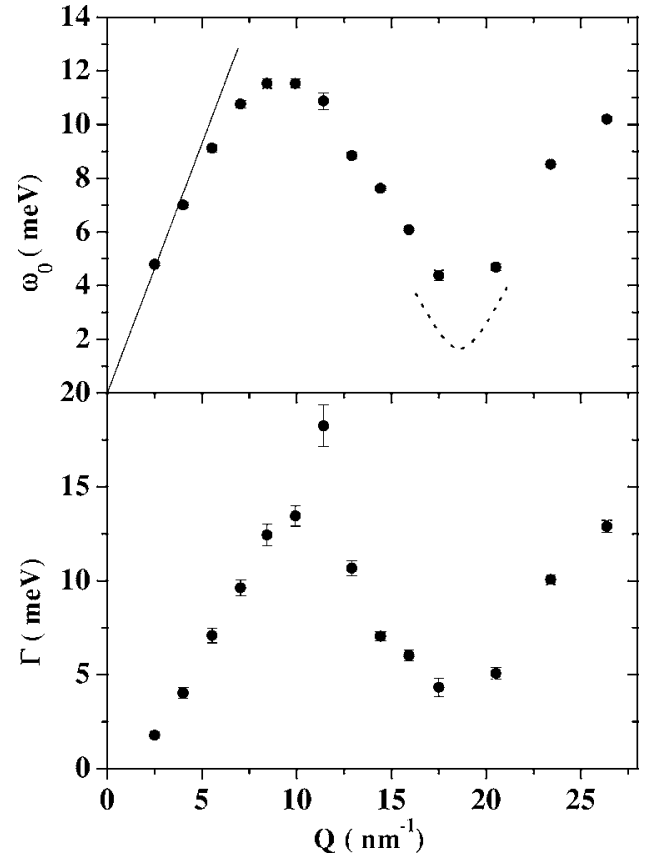

FIG. 4. Dispersion of eigenfrequency $\omega_{0}$ and damping $\Gamma$ of the DHO ansatz [see Eq. (1)] for $B$ DNA at ambient temperature. The initial slope, corresponding to a sound velocity of $2.84 \mathrm{~km} / \mathrm{sec}$, is indicated by the thin, straight line. The dotted hyperbola represents results obtained by neutron scattering [26].

damping $\Gamma$ on the basis of two simplified models, i.e., the modes of a simplified double helix and the effect of disorder on the dispersion of a linear chain.

A model for the external modes of an idealized double helix has been discussed by Capellman and Biem [44]. Here, the nucleotide is reduced to a mass point, a twofold axis $C_{2 z}$ creates the "base pair," and a tenfold screw axis $S_{10 z}$ transforms it into a double helix with the helix pitch of $3.38 \mathrm{~nm}$ for the $B$ conformation. In the extended zone scheme, this model has then 6 dispersion branches corresponding to the 6 degrees of freedom of the dumbbell representing the base pair. The fact that $C_{2 z}$ conserves the wave vector leads to a grouping of those 6 modes into two noninteracting tripletsone in which both strands of the double helix move in phase (ip modes) and one where the two strands are displaced oppositely (oop modes).

The center of mass of the average nucleotide (base+sugar + phosphate) for the mass point results in a distance of $0.6 \mathrm{~nm}$ from the helical axis and a mass of about $320 \mathrm{amu}$ $\left(0.531 \times 10^{-21} \mathrm{~g}\right)$. This next-neighbor $(\mathrm{NN})$ force model contains 4 force parameters which are, however, not arbitrary. They can be related approximately to (for example) the maximum frequency $\omega_{0}$ and the speeds of the 3 acoustic modes (longitudinal $v_{\mathrm{LA}}$, transverse $v_{\mathrm{TA}}$, and torsional $v_{\mathrm{RA}}$ ). From our measurements we estimate $\omega_{0} \approx 14 \mathrm{meV}$ and $v_{\mathrm{LA}} \approx 2900 \mathrm{~m} / \mathrm{s}$. The recent determination of the torsional modulus $C=410 \mathrm{pN} \mathrm{nm}^{2}$ by Bryant et al. [45] corresponds to $v_{\mathrm{RA}} \approx 0.6 \mathrm{~km} / \mathrm{sec}$ for the above moment of inertia of the effective mass point. Stability of the double helix requires $1000 \mathrm{~m} / \mathrm{sec}<v_{\mathrm{TA}}<1400 \mathrm{~m} / \mathrm{s}$. The upper limit leads to an appreciable softening of $v_{\mathrm{RA}}$, the lower limit to instability of

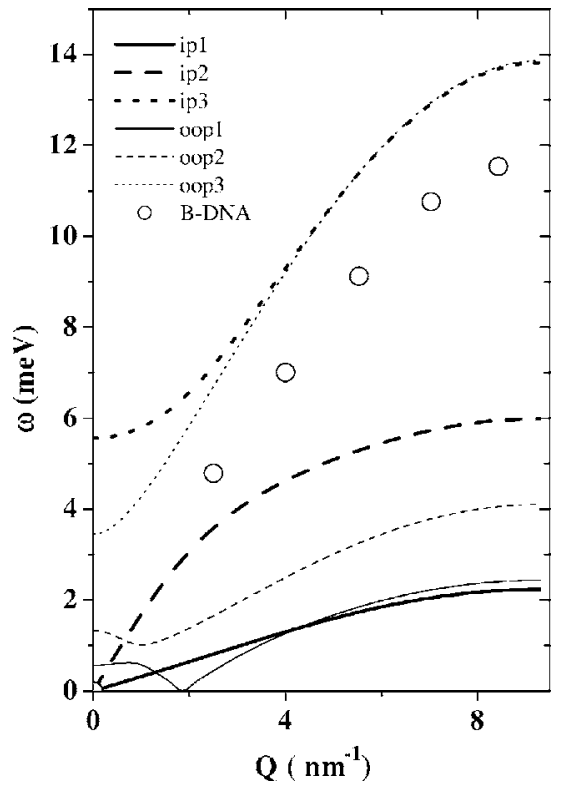

FIG. 5. The $2 \times 3$ dispersion curves of the model for a simplified double helix in the extended zone scheme [44]. The model parameters are described in the text. For the triplet of ip modes (thick lines), both strands move in phase whereas they move with different sign for the oop modes (thin lines). The experimental results for $B$ DNA at ambient temperature are shown by open circles.

the antiphase motion of the strands along the helical axis. We choose $v_{\mathrm{TA}}=1100 \mathrm{~m} / \mathrm{s}$ which delivers $v_{\mathrm{LA}}=2650 \mathrm{~m} / \mathrm{s}$ and $v_{\mathrm{RA}}=480 \mathrm{~m} / \mathrm{s}$. The resulting six dispersion curves are shown in Fig. 5 together with the experimental data for $B$ DNA at ambient temperature. For infinite wavelengths $(Q \rightarrow 0)$, the three "in-phase"- or ip-modes (thick lines) correspond to displacements along $\varphi, z^{-}$, and $\rho$ direction of a cylindrical coordinate system $(z \|, \rho \perp$ helical axis). Thus ip1 starts as torsional mode, ip2 as LA mode, and ip3 as breathing mode at low $Q$ values. Because of the helical shape, the TA branches start at a phase of $\pi / 5$ or $Q \cong \pi / 5 / 0.338 \mathrm{~nm}^{-1} \cong 1.86 \mathrm{~nm}^{-1}$. For one strand, the displacement is inverted for the three "out-of phase" or oop modes (thin lines). The extent to which these modes might contribute to the measured dispersion can be demonstrated by considering the dynamical structure factor $F_{\text {dyn }}$ for $Q$ along the helical axis $z$

$$
F_{\text {dyn }} \propto e^{-u^{2} Q^{2}} Q \sum_{k=1}^{20} \zeta_{k} e^{i Q z_{k}} .
$$

Here, $\zeta_{k}$ and $z_{k}$ denote the $z$ components of eigenvector and position of mass point $k$, respectively. A mean squared displacement of $u^{2}=5 \times 10^{-4} \mathrm{~nm}^{2}$ was assumed. Apart from a constant factor, the intensity $I$ of a mode is then given by $I=\left|F_{\text {dyn }}\right|^{2} / \omega_{0}^{2}$. Obviously, the oop modes are "invisible" because of the opposite displacements $\zeta_{k}$ and identical positional coordinate $z_{k}$ for a base pair. Note, that this is no longer true for the inclined base pair when also all six modes may interact (loss of $C_{2 z}$ axis). The dispersion and the intensity, multiplied by the eigenvalue for the three remaining ip modes, are shown in Fig. 6, where the intensity is proportional to the symbol size. It is evident that the 


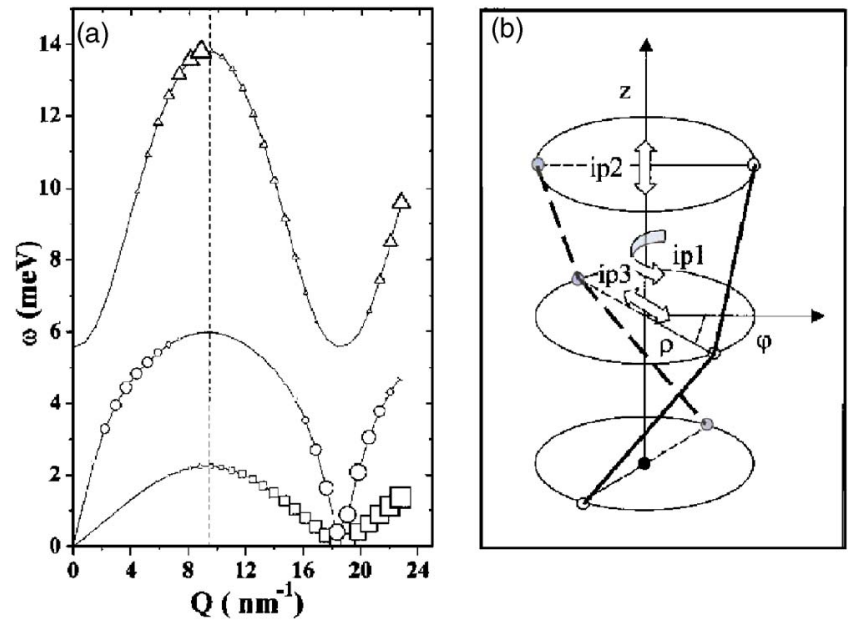

FIG. 6. (Color online) Left panel: Calculated dispersion and intensity for the model of a simplified double helix in the extended zone scheme [44]. From lower to higher frequency, the three branches correspond to torsional (ip1), compressional (ip2), and breathing (ip3) motion at infinite wavelength. Note that for clarity of presentation the intensity has been multiplied by the eigenvalue, and is proportional to the symbol size. Right panel: Schematic representation of the DNA. Each base pair is characterized by its radius $\rho$, angle $\varphi$, and height $z$. The arrows indicate approximately the displacement patterns of the three modes, which are visible in the IXS experiment.

dominating intensity arises from the low lying torsional branch. Although its displacement is perpendicular to the helical direction for $Q \rightarrow 0$, it mixes in rapidly displacement components along this direction for $Q>0$. This intrinsic coupling between motion around and along the helical direction has been clearly demonstrated by the twist and stretch experiments on single DNA molecules [45]. Of course, this branch will become an optical one for a bundle of molecules with interhelical interaction being strongly dependent on humidity and merging into quasielastic scattering at ambient temperature, as observed by Raman scattering [46]. The other two branches - the compressional (LA) and breathing modes-undergo an "anticrossing" in the region $3 \mathrm{~nm}^{-1}<Q<5 \mathrm{~nm}^{-1}$ which obviously leads to an exchange of eigenvectors. Thus, in the first Brillouin zone, the experiment should observe either the LA or the breathing branch. In the second Brillouin zone, however, one might conclude that the experimentally determined DHO parameters represent an effective average over the response due to the LA and breathing branch. On the other hand, within experimental resolution, we observe neither an indication of a bimodal spectral response nor a deviation from the effective sinusoidal dispersion for low $Q$ values. In this context it seems worth noting that the breathing modes in particular stress the anharmonic Morse potential for the hydrogen bonding between bases $[17,18,47]$.

The second model includes the effect of disorder on the dispersion of a linear chain. As a matter of fact, native DNA is by construction disordered due to the sequence of adeninethymine and guanine-cytosine base pairs. Thus, strictly speaking, plane wave excitations are no longer eigenstates and become damped even in the harmonic approximation.

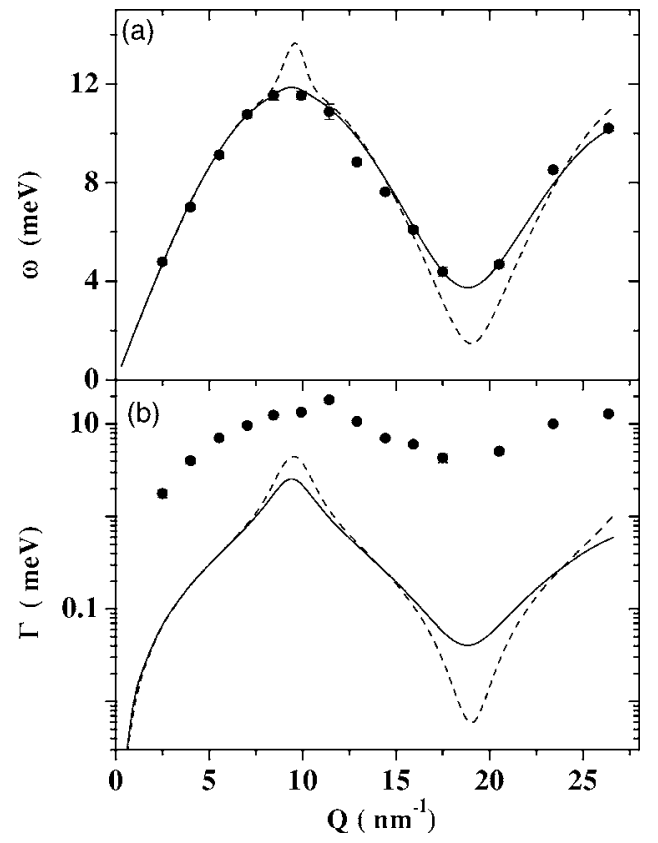

FIG. 7. Predictions for frequency (a) and damping (b) of phononlike excitations of a disordered linear chain by the coherent potential and quasiparticle approximation $[48,49]$. Disorder is represented by the relative variances $\sigma$ (NN distance) and $\lambda$ ( $\mathrm{NN}$ force/ mass). Dashed lines correspond to $\lambda=0.03$ and the experimentally observed $\sigma=0.036$. The solid lines are obtained by tripling this value of $\sigma$. Solid symbols show the fitted values for $B$ DNA at ambient temperature.

The order of magnitude of this damping may be estimated in the coherent potential [48] and quasicrystalline approximation [49] applied to an effective linear chain with NN interaction. A rectangular shape is assumed for the force-constant/ mass distribution (relative variance $=\lambda$ ) and a Gaussian for that of the NN distance (relative variance $=\sigma$ ). From the width of the base pair peak for $B$ DNA (see Fig. 2) follows $\sigma \cong 3.6 \%$ and $\lambda \cong 2 \%$ would result from the mass fluctuation of the base pairs, alone. A value of $\lambda=3 \%$ is assumed for the calculation together with an average NN distance of $0.34 \mathrm{~nm}$ and an average speed of sound of $3000 \mathrm{~m} / \mathrm{s}$. Figure 7 shows the comparison of this quasicrystalline approximation for two values of $\sigma$ with the DHO parameters for $B$ DNA. The calculation, using $\lambda \cong 3 \%$ and $\sigma \cong 10 \%$ nicely describes the experimental dispersion curve for $B$ DNA including its minimum around $18.6 \mathrm{~nm}^{-1}$. Adopting the information from the $S(Q)$ scan with $\sigma \cong 3.6 \%$ would correspond closely to the neutron measurements (see Fig. 4). The appropriate value is, however, of secondary importance. The essential point is that the damping parameter due to this disorder is by one order of magnitude smaller than the observed one. This discrepancy persists even for a variance $\lambda$ as large as $10 \%$ where the limit of the DHO description is reached.

\section{RESULTS FOR THE $A, C$, AND $D$ CONFORMATION AND DISCUSSION}

Figure 8 shows the resulting dispersion and the excitation width for the other three DNA conformations at ambient 


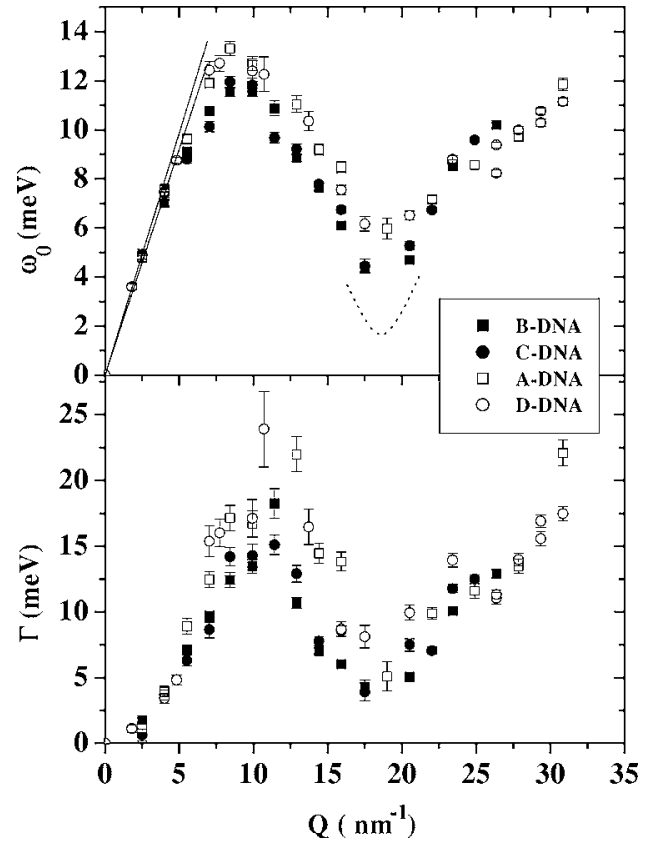

FIG. 8. Dispersion of eigenfrequency $\omega_{0}$ and damping $\Gamma$ of the DHO ansatz [see Eq. (2)] resulting from the four datasets of the $A$, $B, C, D$ DNA samples at ambient temperature. The initial slopes corresponding to sound velocities of 2.8 and $3.0 \mathrm{~km} / \mathrm{sec}$ are indicated by the two thin, straight lines. The dotted hyperbola represents results obtained by neutron scattering [26].

temperature, together with the already reported one of the $B$ conformation. Despite large differences in their static structure, as manifested in the recorded $S(Q)$ spectra (see Fig. 2), the dispersion shape as well as the $Q$ evolution of the damping are very similar. It seems that $C$ DNA resembles $B$ DNA, as does $A$ DNA and $D$ DNA, and that there are slight differences between these two pairs. This mainly concerns the maximum of the excitation energy (higher for $A$ and $D$ DNA), whereas the minimum around $Q_{0}$ is only pronounced for the $B$ conformation, as expected from the very sharp and intense peak in the $S(Q)$ spectrum. Table I summarizes the derived sound velocities as well as the result of a power law fit to the excitation width for the $Q$ range between 2.5 and $10 \mathrm{~nm}^{-1}$.

The similarity of the four dispersion curves and the $Q$ evolution of the excitation width points towards a highfrequency dynamics, which is only weakly dependent on the structural details. We nevertheless observe, as pointed out above, that the phonon dispersion of the $B$ conformation, which can be considered the most "ordered" form of DNA,

TABLE I. Sound velocities, $v$, and $Q$ dependence of sound damping, $\Gamma(Q)$ for $A, B, C$, and $D$ DNA at ambient temperature.

\begin{tabular}{clc}
\hline \hline Conformation & \multicolumn{1}{c}{$v[\mathrm{~m} / \mathrm{s}]$} & $\Gamma[\mathrm{meV}]$ \\
\hline$A$ & $2880 \pm 100$ & $0.98 \times Q^{1.27}$ \\
$B$ & $2830 \pm 50$ & $1.04 \times Q^{1.30}$ \\
$C$ & $2925 \pm 100$ & $0.87 \times Q^{1.33}$ \\
$D$ & $2985 \pm 50$ & $0.87 \times Q^{1.28}$ \\
\hline \hline
\end{tabular}

displays the most pronounced minimum around the $Q$ position of the first maximum in the $S(Q)$. On the other hand, the dominating effect appears to be the structural disorder induced by the random sequence of the base pairs. A further contribution to the disorder arises from the limited conformational homogeneity, at least for $A, B$, and $D$ DNA. It is important to emphasize that our two model calculations for the $B$ conformation cannot be brought into quantitative agreement with the experimental observations, in particular concerning the strong damping of the modes. This indicates that crystallinelike approaches to describe the collective modes, even if disorder is included, are not appropriate. Numerous IXS studies on liquid systems have yielded a qualitatively similar $Q$ evolution of the phononlike excitations and their width $[41,50]$. It seems therefore more appropriate to use a formalism, which abandons the concept of plane wave normal modes with an infinite correlation length. One possible approach starts from a randomly chosen configuration at thermodynamic equilibrium, for which the equations of motion can be linearized, defining some instantaneous normal mode (INM) and their corresponding relaxation times and frequencies [20]. These quantities are then averaged over the initial configuration chosen from the Gibbs ensemble. The resulting normal modes are disordered and have a finite correlation length, giving therefore rise to broad excitations. This approach has been employed by Cocco and Monasson for $B$ DNA in solution [19]. They find that the normal modes are highly disordered and coherent over a few base pairs only, in good agreement with our results.

\section{SUMMARY}

Probing oriented DNA by inelastic x-ray scattering has provided a survey of the spectral response covering the external modes $(-30<\hbar \omega<30 \mathrm{meV})$ and ranging from the long wavelength limit up to the region of umklapp excitations $\left(2.5<\mathbf{Q}<30 \mathrm{~nm}^{-1}\right)$. All four tested conformations exhibit liquidlike dispersion, i.e., strong damping and a minimum around the inverse stacking distance of base pairs along the helical axis. This observation demonstrates that the group velocity derived from umklapp phonons as observed by neutron scattering is much lower than the speed of sound at long wavelengths due the effect of disorder and anharmonicity $[23,24]$. The maximum restoring forces are found to be about $30 \%$ larger for conformations with shorter helical pitch $(A$ DNA and $D$ DNA) as compared to the $B$ or $C$ conformations.

The limits of interpreting the observed large damping parameters in terms of either unresolved dispersion branches or disorder within the quasicrystalline approximation have been shown by quantifying two simple model approaches. As a consequence, the experimental data suggest the inclusion of both strong anharmonicity and finite coherence length of modes in modeling the low frequency excitations of DNA. It is tempting to associate the large damping with the strong anharmonicity of the effective Morse potential of the hydrogen bonds between nucleic acid pairs. An extension of the modeling approach of Cocco and Monasson [19] to all dominating external degrees of freedom seems highly desirable. 


\section{ACKNOWLEDGMENTS}

The authors thank Dr. J. Granzin from the Research Center Juelich (IBI 2) for providing the fiber diffraction patterns of the samples used. We are grateful to Francesco
Sette and Bruno Dorner for their contribution in the beginning of the project. Denis Gambetti and Christian Henriquet are acknowledged for the design and the preparation of the sample cell and its environment. The work was supported by the Swedish Medical Research Council.
[1] W. Fuller, M. H. F. Wilkins, H. R. Wilson, L. D. Hamilton, S. Arnott, J. Mol. Biol. 12, 60 (1965).

[2] R. Langridge, D. A. Marvin, W. E. Seeds, H. R. Wilson, C. W. Hooper, and M. H. F. Wilkins, J. Mol. Biol. 2, 38 (1960).

[3] D. A. Marvin, M. Spencer, M. H. F. Wilkins, and L. D. Hamilton, J. Mol. Biol. 3, 547 (1961).

[4] D. R. Davies and R. L. Baldwin, J. Mol. Biol. 6, 251 (1963).

[5] S. Arnott, R. Chandrasekaran, D. L. Birdsall, A. G. Leslie, and R. L. Ratliff, Nature (London) 283, 743 (1980).

[6] A. H.-J. Wang, G. J. Quigley, F. J. Kolpak, J. L. Crawford, J. H. van Boom, G. van der Marel, and A. Rich, Nature (London) 282, 680 (1979).

[7] M. W. Shotton, L. H. Pope, V. T. Forsyth, R. C. Denny, J. Archer, P. Langan, H. Ye, and C. Boote, J. Appl. Crystallogr. 31, 758 (1998).

[8] W. Fuller, V. T. Forsyth, and A. Mahendrasingam, Philos. Trans. R. Soc. London 359 1448, 1237 (2004).

[9] C. Reiss, Nonlinear Excitations in Biomolecules, edited by M. Peyrard, Les Editions de Physique (Springer, Berlin 1994), p. 29.

[10] A. Mahendrasingam, V. T. Forsyth, R. Hussain, R. J. Greenall, W. J. Pigram, and W. Fuller, Science 233, 195 (1986).

[11] J. M. Eyster and E. W. Prohofsky, Phys. Rev. Lett. 38, 371 (1977)

[12] W. Cochran, Adv. Phys. 9, 387 (1960).

[13] Y. Kim, K. V. Devi-Prasad, and E. W. Prohofsky, Phys. Rev. B 32, 5185 (1985).

[14] V. V. Prabhu, L. Young, and E. W. Prohofsky, Phys. Rev. B 39, 5436 (1989).

[15] S. W. Englander, N. R. Kallenbach, A. J. Heeger, J. A. Krummhansl, and S. Litwin, Proc. Natl. Acad. Sci. U.S.A. 77, 7222 (1980).

[16] E. W. Prohofsky, Phys. Rev. A 38, 1538 (1988).

[17] M. Peyrard and A. R. Bishop, Phys. Rev. Lett. 62, 2755 (1989).

[18] T. Dauxois and M. Peyrard, Phys. Rev. E 51, 4027 (1995).

[19] S. Cocco and R. Monasson, J. Chem. Phys. 112, 10017 (2000).

[20] T. Keyes, J. Phys. Chem. A 101, 2921 (1997).

[21] G. Maret, R. Oldenbourg, G. Winterling, K. Dransfeld, and A. Rupprecht, Colloid Polym. Sci. 257, S1017 (1979).

[22] M. B. Hakim, S. M. Lindsay, and J. Powell, Biopolymers 23, 1185 (1984).

[23] N. J. Tao and S. M. Lindsay, Biopolymers 26, 171 (1987).

[24] S. A. Lee, S. M. Lindsay, J. W. Powell, T. Weidlich, N. J. Tao, and G. D. Lewen, Biopolymers 26, 1637 (1987).

[25] Y. Tominaga, M. Shida, K. Kubota, H. Urabe, Y. Nishimura, and M. Tsuboi, J. Chem. Phys. 83 11, 5972 (1985).

[26] H. Grimm, H. Stiller, C. F. Majkrzak, A. Rupprecht, and U. Dahlborg, Phys. Rev. Lett. 59, 1780 (1987).

[27] H. Grimm and A. Rupprecht, Physica B 234-236, 183 (1997).

[28] L. van Hove, Phys. Rev. 95, 249 (1954).

[29] For the remainder of the paper, we assume for simplicity $\hbar$ $=1$.

[30] Y. Liu, D. Berti, A. Faraone, W.-R. Chen, A. Atalas, H. Sinn, E. Alp, A. Said, P. Baglioni, and S.-H. Chen, Phys. Chem. Chem. Phys. 6, 1499 (2004).

[31] H. Grimm, P. M. Gehring, S. M. Shapiro, R. Kahn, and A. Rupprecht, Physica B 213, 780 (1995).

[32] C. Liao and S. H. Chen, Phys. Rev. E 64, 021205 (2001).

[33] L. H. Pope, M. W. Shotton, V. T. Forsyth, D. Hughes, R. C. Denny, and W. Fuller, Biophys. Chem. 70, 161 (1998).

[34] A. Rupprecht, Acta Chem. Scand. (1947-1973) 20, 494 (1966).

[35] A. Rupprecht and B. Forslind, Biochim. Biophys. Acta 204, 304 (1970).

[36] H. Grimm and A. Rupprecht, Physica B 174, 291 (1991).

[37] H. Grimm and A. Rupprecht, Eur. Biophys. J. 17, 173 (1989).

[38] R. Verbeni, F. Sette, M. H. Krisch, U. Bergmann, B. Gorges, C. Halcoussis, K. Martel, C. Masciovecchio, J. F. Ribois, G. Ruocco, and H. Sinn, J. Synchrotron Radiat. 3, 62 (1996).

[39] C. Masciovecchio, U. Bergmann, M. Krisch, G. Ruocco, F. Sette, and R. Verbeni, Nucl. Instrum. Methods Phys. Res. B 111, 181 (1996).

[40] C. Masciovecchio, U. Bergmann, M. Krisch, G. Ruocco, F. Sette, and R. Verbeni, Nucl. Instrum. Methods Phys. Res. B 117, 339 (1996).

[41] G. Ruocco and F. Sette, J. Phys.: Condens. Matter 11, R259 (1999).

[42] E. Burkel, Rep. Prog. Phys. 63, 171 (2000).

[43] H. Grimm, A. P. Sokolov, and A. J. Dianoux, Appl. Phys. A 74, 1248 (2002).

[44] H. Capellmann and W. Biem, Z. Phys. 209, 276 (1968).

[45] Z. Bryant, M. D. Stone, J. Gore, S. B. Smith, N. R. Cozzarelli, and C. Bustamante, Nature (London) 424, 338 (2003).

[46] H. Urabe, H. Hayashi, Y. Tominaga, Y. Nishimura, K. Kubota, and M. Tsuboi, J. Chem. Phys. 83, 5972 (1985).

[47] M. V. Sataric and J. A. Tuszynski, Phys. Rev. E 65, 051901 (2002).

[48] I. Ishida and T. Matsubara, J. Phys. Soc. Jpn. 38, 534 (1975).

[49] P. Kleinert and R. Leihkauf, Phys. Status Solidi B 97, 491 (1980).

[50] T. Scopigno, G. Ruocco, and F. Sette, Rev. Mod. Phys. 77, 881 (2005). 